\title{
Biomimetic Synthesis of Elysiapyrones A and B
}

\author{
Jennifer E. Barbarow, Aubry K. Miller and Dirk Trauner* \\ Center for New Directions in Organic Synthesis, Department of Chemistry, University of \\ California-Berkeley, Berkeley, California 94720

\section{Supporting Information}

Unless otherwise noted, all reagents were purchased from commercial suppliers and used without further purification. Melting points were measured on a Büchi melting point apparatus and are uncorrected. ${ }^{1} \mathrm{H}$ - and ${ }^{13} \mathrm{C}$-NMR spectra were recorded on a Bruker DRX 500 or $\mathrm{AV}-300$ spectrometer in $\mathrm{CDCl}_{3}$. Infrared spectra (IR) were obtained on $\mathrm{NaCl}$ plates with an ATI Mattson Gemini FTIR spectrometer. High-resolution mass spectra (HRMS) were obtained on a VG ProSpec Mass Spectrometer using electron impact (EI) at $70 \mathrm{eV}$.

Unless otherwise noted, all reaction mixtures were magnetically stirred in oven-dried glassware under a blanket of argon. External bath temperatures were used to record all reaction mixture temperatures. Analytical thin layer chromatography (TLC) was carried out on Merck silica gel $60 \mathrm{~F}_{254}$ TLC plates. TLC visualization was accomplished using $254 \mathrm{~nm}$ UV light or charring solutions of anisaldehyde, $\mathrm{KMnO}_{4}$ and cerric ammonium molybdenate. Flash chromatography was performed on ICN siliTech 32-63 D $60 \AA$ silica gel according to the procedure of Still. ${ }^{1}$

Tetrahydrofuran (THF) and dichloromethane (DCM) were dried according to the procedure described by Bergman. ${ }^{2}$ Benzene was distilled from $\mathrm{CaH}_{2}$ immediately prior to use. DMF was dried over $4 \AA$ molecular sieves. $n$-Butyl lithium (n-BuLi) was titrated using diphenylacetic acid in THF. CuI was purified by precipitation from hot aqueous NaI. Extracts were dried over anhydrous $\mathrm{MgSO}_{4}$ and solvents were removed with a rotary evaporator at aspirator pressure.

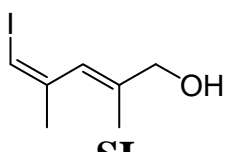

SI

(2E, 4Z)-5-iodo-2,4-dimethylpenta-2,4-dien-1-ol (S1): To a solution of $\mathbf{9}^{3}$ (1.00 g, 3.57 mmol) in DCM $(35 \mathrm{~mL})$ was added a solution of DIBAH $(6.0 \mathrm{~mL}, 9.0 \mathrm{mmol}, 1.5 \mathrm{M}$ in toluene) at $-78{ }^{\circ} \mathrm{C}$ under argon. After $45 \mathrm{~min}$, the reaction mixture was quenched with half saturated Rochelle's salt $(100 \mathrm{~mL})$, diluted with DCM $(50 \mathrm{~mL})$ and stirred vigorously for 30 min. The two layers were separated and the aqueous layer was extracted with DCM $(2 \times 30$ $\mathrm{mL}$ ). The combined organic layers were dried, filtered, concentrated in vacuo, and purified by column chromatography (20\% EtOAc in hexanes) to provide $836 \mathrm{mg}(98 \%)$ of S1 as a colorless oil: $\mathrm{R}_{f} 0.46$ (30\% EtOAc in hexanes); IR (thin film) 3320(br), 2909, $2855 \mathrm{~cm}^{-1} ;{ }^{1} \mathrm{H}$ NMR $(500 \mathrm{MHz}): \delta_{\mathrm{H}} 6.03(\mathrm{~s}, 1 \mathrm{H}), 5.93(\mathrm{~s}, 1 \mathrm{H}), 4.07(\mathrm{~s}, 2 \mathrm{H}), 1.98(\mathrm{~s}, 3 \mathrm{H}) 1.71(\mathrm{~s}, 3 \mathrm{H}) ;{ }^{13} \mathrm{C}$ NMR $(125 \mathrm{MHz}): \delta_{\mathrm{C}} 144.5,139.1,126.7,77.6,68.3,24.7,15.6$; HRMS calcd for $\mathrm{C}_{7} \mathrm{H}_{11} \mathrm{IO}$ $\left(\mathrm{M}^{+}\right)$: 237.9902; found: 237.9857 . 


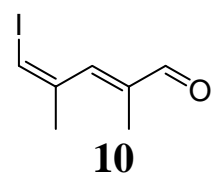

(2E, 4Z)-5-iodo-2,4-dimethylpenta-2,4-dienal (10): To a solution of S1 (830 mg, $3.49 \mathrm{mmol}$ ) in DCM taken directly from a squirt bottle $(35 \mathrm{~mL})$ was added the Dess-Martin periodinane (DMP) $(1.65 \mathrm{~g}, 3.89 \mathrm{mmol})$ at $0{ }^{\circ} \mathrm{C}$ open to the air. After addition, the reaction mixture was allowed to warm to rt. After $1 \mathrm{~h}$, a mixture of 1:1:1 $\mathrm{H}_{2} \mathrm{O}$ : saturated aqueous $\mathrm{NaHCO}_{3}$ : saturated aqueous $\mathrm{Na}_{2} \mathrm{~S}_{2} \mathrm{O}_{3}(50 \mathrm{~mL})$ was added and the two layers were separated. The aqueous layer was extracted with DCM $(2 \times 20 \mathrm{~mL})$ and the combined organic layers were dried, filtered, and concentrated in vacuo. The product was purified by column chromatography ( $5 \% \mathrm{Et}_{2} \mathrm{O}$ in hexanes) to provide $680 \mathrm{mg}(83 \%)$ of $\mathbf{1 0}$ as a colorless oil: $\mathrm{R}_{f}$ $0.35\left(5 \% \mathrm{Et}_{2} \mathrm{O}\right.$ in hexanes); IR (thin film) $1681 \mathrm{~cm}^{-1} ;{ }^{1} \mathrm{H}$ NMR $(500 \mathrm{MHz}): \delta_{\mathrm{H}} 9.52(\mathrm{~s}, 1 \mathrm{H})$, 6.90 (s, $1 \mathrm{H}), 6.47$ (s, $1 \mathrm{H}), 2.14(\mathrm{~s}, 3 \mathrm{H}) 1.86(\mathrm{~s}, 3 \mathrm{H}) ;{ }^{13} \mathrm{C} \mathrm{NMR}(125 \mathrm{MHz}): \delta_{\mathrm{C}} 195.5,150.5$, 142.4, 140.1, 85.3, 24.0, 10.9; HRMS calcd for $\mathrm{C}_{7} \mathrm{H}_{9} \mathrm{IO}\left(\mathrm{M}^{+}\right)$: 235.9698; found: 235.9692 .

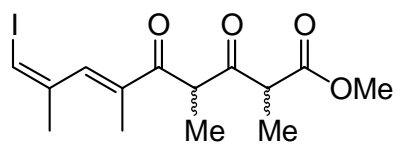

12

(6E, 8Z)-methyl-9-iodo-2,4,6,8-tetramethyl-3,5-dioxonona-6,8-dienoate (12): To a suspension of $\mathrm{NaH}(233 \mathrm{mg}, 5.82 \mathrm{mmol})$ in THF $(10 \mathrm{~mL})$ was added a solution of $\mathbf{1 1}(840 \mathrm{mg}$, $5.83 \mathrm{mmol})$ in THF ( $8 \mathrm{~mL}+$ two $1 \mathrm{~mL}$ rinses) at $0{ }^{\circ} \mathrm{C}$ under argon. After $15 \mathrm{~min}$, the mixture was cooled to $-78^{\circ} \mathrm{C}$ and a solution of $n$-BuLi $(2.50 \mathrm{~mL}, 5.62 \mathrm{mmol}, 2.25 \mathrm{M}$ in hexanes) was added. After $1 \mathrm{~h}$, the reaction mixture was allowed to warm to $-10{ }^{\circ} \mathrm{C}$ for $10 \mathrm{~min}$ and was then cooled to $-78{ }^{\circ} \mathrm{C}$. To this mixture was added a solution of $\mathbf{1 0}(605 \mathrm{mg}, 2.56 \mathrm{mmol})$ in THF $(8$ $\mathrm{mL}+$ two $1 \mathrm{~mL}$ rinses). After $45 \mathrm{~min}$, the reaction was quenched with half saturated aqueous $\mathrm{NH}_{4} \mathrm{Cl}(100 \mathrm{~mL})$ and diluted with $\mathrm{Et}_{2} \mathrm{O}(50 \mathrm{~mL})$. The two layers were separated and the organic layer was washed with $\mathrm{H}_{2} \mathrm{O}(50 \mathrm{~mL})$ and brine $(50 \mathrm{~mL})$. The organic layer was dried, filtered, and concentrated in vacuo. The products were purified by column chromatography (35\% $\mathrm{Et}_{2} \mathrm{O}$ in hexanes) to give $761 \mathrm{mg}(78 \%)$ of a mixture of diastereomers that were taken on without full characterization. To a solution of this mixture of diastereomers $(785 \mathrm{mg}, 2.06$ mmol) in DCM taken directly from a squirt bottle $(15 \mathrm{~mL})$ was added DMP $(1.005 \mathrm{~g}, 2.37$ $\mathrm{mmol}$ ) at $0{ }^{\circ} \mathrm{C}$ open to the air. After addition, the reaction mixture was allowed to warm to $\mathrm{rt}$. After 40 min, a mixture of 1:1:1 $\mathrm{H}_{2} \mathrm{O}$ : saturated aqueous $\mathrm{NaHCO}_{3}$ : saturated aqueous $\mathrm{Na}_{2} \mathrm{~S}_{2} \mathrm{O}_{3}$ $(20 \mathrm{~mL})$ was added and the two layers were separated. The aqueous layer was extracted with DCM $(2 \times 10 \mathrm{~mL})$ and the combined organic layers were dried, filtered, and concentrated in vacuo. The products were purified by column chromatography (15\% EtOAc in hexanes) to provide $673 \mathrm{mg}(86 \%)$ of 12 as a colorless oil that was a mixture of inconsequential diastereomers: $\mathrm{R}_{f} 0.33$ (20\% EtOAc in hexanes); LRMS(EI) $\left(\mathrm{M}^{+}\right): 378$. 


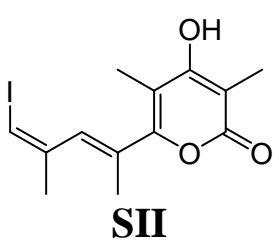

4-hydroxy-6-((2E, 4Z)-5-iodo-4-methylpenta-2,4-dien-2-yl)-3,5-dimethyl-2H-pyran-2-one (SII): A solution of $12(750 \mathrm{mg}, 1.98 \mathrm{mmol})$ and $\mathrm{DBU}(0.30 \mathrm{~mL}, 2.0 \mathrm{mmol})$ in benzene $(10$ $\mathrm{mL}$ ) was heated to reflux under argon. After $1.5 \mathrm{~h}$ the reaction mixture was concentrated in vacuo. The product was purified by column chromatography (45\% EtOAc in hexanes w/ $0.002 \% \mathrm{AcOH})$ to provide $509 \mathrm{mg}(74 \%)$ of SII as a white solid: $\mathrm{R}_{f} 0.25(45 \%$ EtOAc in hexanes w/ $0.002 \% \mathrm{AcOH}$ ); $\mathrm{mp} 137-138{ }^{\circ} \mathrm{C}$; IR (thin film) $3100,1660,1555 \mathrm{~cm}^{-1} ;{ }^{1} \mathrm{H}$ NMR (500 MHz): $\delta_{\mathrm{H}} 6.98(\mathrm{br} \mathrm{s}, 1 \mathrm{H}), 6.22(\mathrm{~m}, 1 \mathrm{H}), 6.07(\mathrm{~s}, 1 \mathrm{H}), 2.17(\mathrm{~s}, 3 \mathrm{H}) 2.04(\mathrm{~m}, 6 \mathrm{H}) 1.97$ $(\mathrm{d}, J=1.3 \mathrm{~Hz}, 3 \mathrm{H}) ;{ }^{13} \mathrm{C}$ NMR $(125 \mathrm{MHz}): \delta_{\mathrm{C}} 166.0,165.5,158.1,143.8,136.3,130.9,107.8$, 99.7, 79.7, 24.5, 16.8, 12.1, 9.0; HRMS calcd for $\mathrm{C}_{13} \mathrm{H}_{15} \mathrm{IO}_{3}\left(\mathrm{M}^{+}\right)$: 346.0066; found: 346.0066 .

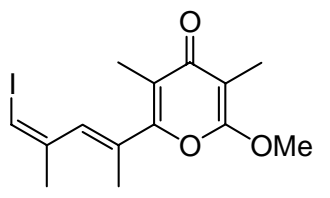

13

2-((2E, 4Z)-5-iodo-4-methylpenta-2,4-dien-2-yl)-6-methoxy-3,5-dimethyl-4H-pyran-4one (13): To a suspension of SII ( $480 \mathrm{mg}, 1.39 \mathrm{mmol})$ in DCM $(8 \mathrm{~mL})$ was added $\mathrm{MeOSO}_{2} \mathrm{~F}$ $(2.20 \mathrm{~mL}, 27.5 \mathrm{mmol})$ under argon at rt. The reaction flask was wrapped in foil. After $20 \mathrm{~h}$, the solvent was removed on the high-vac in the hood to avoid exposure to $\mathrm{MeOSO}_{2} \mathrm{~F}$. The residue was then redissolved in DCM and the solvent was removed in the same manner twice. The crude material was then dissolved in EtOAc $(20 \mathrm{~mL})$ and washed with $1 \mathrm{~N} \mathrm{NaOH}(10$ $\mathrm{mL}), \mathrm{H}_{2} \mathrm{O}(10 \mathrm{~mL})$, and then brine $(10 \mathrm{~mL})$. The organic layer was dried, filtered, concentrated in vacuo and purified by column chromatography (30\% EtOAc in hexanes) to provide $372 \mathrm{mg}$ (75\%) of $\mathbf{1 3}$ as a white solid: $\mathrm{R}_{f} 0.25$ (30\% EtOAc in hexanes); $\mathrm{mp}$ 102-104 ${ }^{\circ} \mathrm{C}$; IR (thin film) $1655,1587 \mathrm{~cm}^{-1}$; ${ }^{1} \mathrm{H}$ NMR $(500 \mathrm{MHz}): \delta_{\mathrm{H}} 6.26(\mathrm{~s}, 1 \mathrm{H}), 6.17(\mathrm{~s}, 1 \mathrm{H}), 3.98$ $(\mathrm{s}, 3 \mathrm{H}), 2.11(\mathrm{~s}, 3 \mathrm{H}) 2.07(\mathrm{~s}, 3 \mathrm{H}) 2.00(\mathrm{~s}, 3 \mathrm{H}), 1.87(\mathrm{~s}, 3 \mathrm{H}) ;{ }^{13} \mathrm{C}$ NMR $(125 \mathrm{MHz}): \delta_{\mathrm{C}} 181.5$, $162.1,157.5,143.5,136.9,130.5,118.8,99.7,80.0,55.5,24.5,16.7,12.3,7.1$; HRMS calcd for $\mathrm{C}_{14} \mathrm{H}_{17} \mathrm{IO}_{3}\left(\mathrm{M}^{+}\right)$: 360.0222 ; found: 360.0223 .

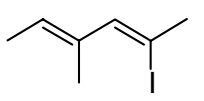

15

(2Z, 4E)-2-Iodo-4-methylhexa-2,4-diene (15): To a solution of (ethyl)triphenylphosphonium iodide $(24.85 \mathrm{~g}, 59.4 \mathrm{mmol})$ in THF $(125 \mathrm{~mL})$ was added $n-\mathrm{BuLi}(27.7 \mathrm{~mL}$ of a $2.15 \mathrm{M}$ solution in hexanes, $59.4 \mathrm{mmol})$. The mixture was stirred for 20 minutes and then cannulated into a solution of $\mathrm{I}_{2}(15.09 \mathrm{~g}, 59.4 \mathrm{mmol})$ in THF $(125 \mathrm{~mL})$ at $-78^{\circ} \mathrm{C}$. The reaction mixture became viscous, opaque, and bright yellow. The temperature was allowed to rise to $-45^{\circ} \mathrm{C}$, and NaHMDS ( $27.8 \mathrm{~mL}$ of a $2.0 \mathrm{M}$ solution in THF, $55.6 \mathrm{mmol}$ ) was added. The mixture turned a deep red color. Stirring was continued for 5 minutes. Then trans-2-methyl-2-butenal 
$(2.50 \mathrm{~g}, 29.7 \mathrm{mmol})$ was added to the solution neat. The mixture turned black, and was allowed to stir for 20 minutes while warming to rt. Aqueous saturated ammonium chloride solution $(500 \mathrm{~mL})$ was added and the reaction mixture was extracted with EtOAc $(2 \times 200$ $\mathrm{mL})$. The combined organic layers were washed with brine $(2 \times 100 \mathrm{~mL})$, dried and concentrated in vacuo. Purification by column chromatography (alumina, $10 \%$ EtOAc in hexanes) gave $2.547 \mathrm{~g}(38.6 \%)$ of $\mathbf{1 5}$ as a yellow oil: $\mathrm{R}_{f} 0.66$ (10\% EtOAc in hexanes); IR (thin film) 2959, 2916, $2870 \mathrm{~cm}^{-1} ;{ }^{1} \mathrm{H}$ NMR $(500 \mathrm{MHz}): \delta_{\mathrm{H}} 5.99(\mathrm{~s}, 1 \mathrm{H}), 5.57(\mathrm{~m}, 1 \mathrm{H}), 2.55$ $(\mathrm{d}, J=1.4 \mathrm{~Hz}, 3 \mathrm{H}), 1.76(\mathrm{~s}, 3 \mathrm{H}), 1.68(\mathrm{~d}, J=6.9 \mathrm{~Hz}, 3 \mathrm{H}) ;{ }^{13} \mathrm{C}$ NMR $(125 \mathrm{MHz}): \delta_{\mathrm{C}} 138.4$, 134.5, 126.5, 96.1, 35.1, 15.7, 13.7; HRMS (EI) calcd for $\mathrm{C}_{7} \mathrm{H}_{11} \mathrm{I}\left(\mathrm{M}^{+}\right)$: 221.990552; found: 221.990343.

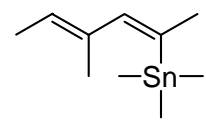

16

(2Z, 4E)-2-trimethylstannyl-4-methylhexa-2,4-diene (16): To a solution of $\mathbf{1 5}$ (1.00 g, 4.5 $\mathrm{mmol})$ in $\mathrm{Et}_{2} \mathrm{O}(25 \mathrm{~mL})$, at $-78{ }^{\circ} \mathrm{C}$, was added $n-\mathrm{BuLi}(2.2 \mathrm{~mL}$ of a $2.15 \mathrm{M}$ solution in hexane, $4.7 \mathrm{mmol})$. After five minutes, trimethyltin chloride $(5.1 \mathrm{~mL}$ of a $1.0 \mathrm{M}$ solution in hexane, 5.1 mmol) was added. The reaction mixture was stirred for one hour and then quenched with $\mathrm{H}_{2} \mathrm{O}$ $(5 \mathrm{~mL})$. The mixture was warmed to $\mathrm{rt}$ and the aqueous layer was extracted with $\mathrm{Et}_{2} \mathrm{O}(5 \mathrm{~mL})$. The combined organic layers were dried with sodium sulfate and concentrated. Purification by column chromatography (alumina, 100\% hexanes) gave $\mathbf{1 6}(915 \mathrm{mg}, \mathbf{7 8 . 5 \%})$ as a clear liquid: $\mathrm{R}_{f} 0.60$ (hexanes); IR (thin film) 2923, 1435, $766 \mathrm{~cm}^{-1} ;{ }^{1} \mathrm{H}$ NMR $(300 \mathrm{MHz}): \delta_{\mathrm{H}} 6.71(\mathrm{~s}, 1 \mathrm{H})$, $5.56(\mathrm{~m}, 1 \mathrm{H}), 2.05(\mathrm{~d}, J=1.7 \mathrm{~Hz}, 3 \mathrm{H}), 1.69(\mathrm{~s}, 3 \mathrm{H}), 1.61(\mathrm{~d}, J=6.8 \mathrm{~Hz}, 3 \mathrm{H}), 0.27(\mathrm{~s}, 9 \mathrm{H})$; ${ }^{13} \mathrm{C}$ NMR $(75 \mathrm{MHz}): \delta_{\mathrm{C}} 145.0,139.2,138.4,121.5,26.5,16.3,13.7,-7.9$; HRMS (EI) calcd for $\mathrm{C}_{9} \mathrm{H}_{17} \mathrm{Sn}\left(\mathrm{M}^{+}-\mathrm{CH}_{3}\right): 245.035225$; found: 245.035381 .

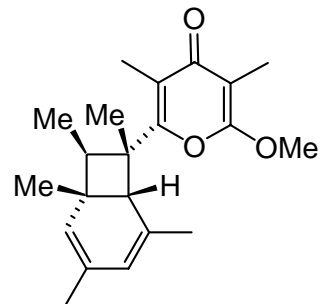

19

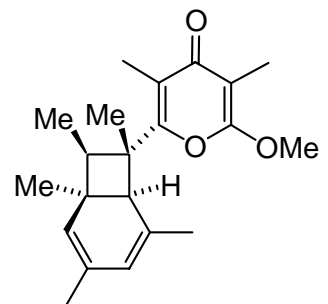

20

Bicyclo [4.2.0]octadienes (19, 20): To a solution of $13(14 \mathrm{mg}, 0.039 \mathrm{mmol})$ and 16 (15 mg, $0.058 \mathrm{mmol})$ in DMF (5 mL) was added tetrakis-triphenyl phosphine palladium (5 mg, 0.0039 mmol), cuprous iodide ( $2 \mathrm{mg}, 0.0078 \mathrm{mmol})$ and cesium fluoride $(12 \mathrm{mg}, 0.078 \mathrm{mmol})$. The reaction mixture was stirred at $40{ }^{\circ} \mathrm{C}$. After $16 \mathrm{~h}$, the solvent was removed in vacuo. Purification by column chromatography (25\% EtOAc in hexanes) and HPLC (15-40\% EtOAc in hexanes over $30 \mathrm{~min}$, Dynamax-60A, Si 83-121-C) afforded $19(5.7 \mathrm{mg}, 44.7 \%)$ as a colorless oil and $20(3.9 \mathrm{mg}, 23.5 \%)$ as a colorless oil.

19: $\mathrm{R}_{f} 0.41$ (50\% EtOAc in hexanes); IR (thin film) $2926,1657,1611 \mathrm{~cm}^{-1} ;{ }^{1} \mathrm{H}$ NMR (500 MHz): $\delta_{\mathrm{H}} 5.43(\mathrm{~s}, 1 \mathrm{H}), 5.01(\mathrm{~s}, 1 \mathrm{H}), 3.96(\mathrm{~s}, 3 \mathrm{H}), 3.16(\mathrm{q}, J=7.3 \mathrm{~Hz}, 1 \mathrm{H}), 2.61(\mathrm{~s}, 1$ H), 1.93 (s, $3 \mathrm{H}), 1.85(\mathrm{~s}, 3 \mathrm{H}), 1.72(\mathrm{~s}, 3 \mathrm{H}), 1.53(\mathrm{~d}, J=1.4 \mathrm{~Hz}, 3 \mathrm{H}), 1.49(\mathrm{~s}, 3 \mathrm{H}), 1.08$ (d, $J$ $=7.5 \mathrm{~Hz}, 3 \mathrm{H}), 0.96(\mathrm{~s}, 3 \mathrm{H}) ;{ }^{13} \mathrm{C} \mathrm{NMR}(125 \mathrm{MHz}): \delta_{\mathrm{C}} 181.9,162.6,162.2,131.1,128.7$, 
127.3, 124.9, 118.8, 99.7, 56.2, 56.1, 52.2, 44.7, 37.3, 24.0, 23.6, 22.0, 21.6, 12.6, 11.5, 7.2; HRMS (EI) calcd for $\mathrm{C}_{21} \mathrm{H}_{28} \mathrm{O}_{3}\left(\mathrm{M}^{+}\right)$: 328.203845; found: 328.203198 .

20: $\mathrm{R}_{f} 0.49$ (50\% EtOAc in hexanes); IR (thin film) $2925,1659,1615 \mathrm{~cm}^{-1} ;{ }^{1} \mathrm{H}$ NMR (500 MHz): $\delta_{\mathrm{H}} 5.54(\mathrm{~s}, 1 \mathrm{H}), 4.90(\mathrm{~s}, 1 \mathrm{H}), 3.25(\mathrm{~s}, 3 \mathrm{H}), 3.08(\mathrm{~s}, 1 \mathrm{H}), 2.43$ (q, J= $7.2 \mathrm{~Hz}, 1$ H), $2.13(\mathrm{~s}, 3 \mathrm{H}), 2.06(\mathrm{~s}, 3 \mathrm{H}), 1.66(\mathrm{~s}, 3 \mathrm{H}), 1.62(\mathrm{~s}, 3 \mathrm{H}), 1.13(\mathrm{~s}, 3 \mathrm{H}), 1.03(\mathrm{~s}, 3 \mathrm{H}), 0.95(\mathrm{~d}$, $J=7.2 \mathrm{~Hz}, 3 \mathrm{H}) ;{ }^{13} \mathrm{C}$ NMR $(125 \mathrm{MHz}): \delta_{\mathrm{C}} 181.2,164.9,162.2,130.8,129.6,125.6,124.2$, $116.9,101.6,57.0,50.4,49.3,47.6,37.9,30.9,23.5,22.2,15.5,10.4,10.3,7.8$; HRMS (EI) calcd for $\mathrm{C}_{21} \mathrm{H}_{28} \mathrm{O}_{3}\left(\mathrm{M}^{+}\right)$: 328.203845; found: 328.203924 .

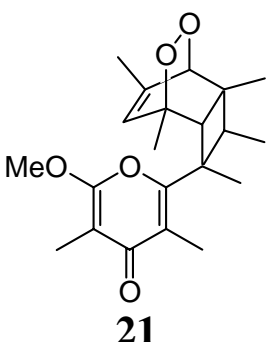

Endo-peroxide (21): In a three-neck flask, fitted with a reflux condenser, methylene blue (2 $\mathrm{mg}, 0.006 \mathrm{mmol})$ was added to a solution of $19(25 \mathrm{mg}, 0.076 \mathrm{mmol})$ in chloroform $(30 \mathrm{~mL})$. Oxygen was bubbled through the solution, and the reaction mixture was stirred under a halogen lamp for $1 \mathrm{~h}$. The solvent was removed in vacuo and purification by column chromatography (50\% EtOAc in hexanes) gave 21 (19 mg, 69.3\%) as a colorless oil; $\mathrm{R}_{f} 0.17$ (50\% EtOAc in hexanes); IR (thin film) 2918, 2359, 1657, $1613 \mathrm{~cm}^{-1} ;{ }^{1} \mathrm{H}$ NMR (500 MHz): $\delta_{\mathrm{H}} 5.70(\mathrm{~s}, 1 \mathrm{H}), 3.96(\mathrm{~d}, J=1.5 \mathrm{~Hz}, 1 \mathrm{H}), 3.92(\mathrm{~s}, 3 \mathrm{H}), 2.91(\mathrm{q}, J=7.5 \mathrm{~Hz}, 1 \mathrm{H}), 2.22(\mathrm{~s}, 1$ H), $2.02(\mathrm{~d}, J=2.0 \mathrm{~Hz}), 1.95(\mathrm{~s}, 3 \mathrm{H}), 1.87(\mathrm{~s}, 3 \mathrm{H}), 1.49(\mathrm{~s}, 6 \mathrm{H}), 1.22(\mathrm{~s}, 3 \mathrm{H}), 1.00(\mathrm{~d}, J=$ $7.5 \mathrm{~Hz}, 3 \mathrm{H}) ;{ }^{13} \mathrm{C}$ NMR $(125 \mathrm{MHz}): \delta_{\mathrm{C}} 181.5,161.9,159.9,142.9,126.3,118.6,114.4,99.8$, 83.1, 77.7, 55.4, 36.9, 36.0, 31.7, 22.8, 21.9, 20.7, 19.2, 14.3, 10.6, 7.1; HRMS (EI) calcd for $\mathrm{C}_{21} \mathrm{H}_{28} \mathrm{O}_{5}\left(\mathrm{M}^{+}\right): 360.193674$; found: 360.194043 .

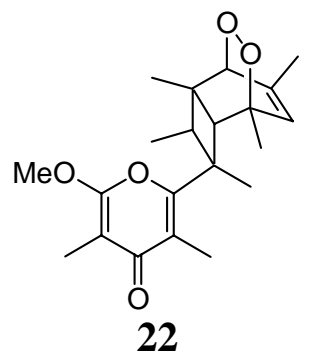

Endo-peroxide (22): In a three-neck flask, fitted with a reflux condenser, methylene blue (2 $\mathrm{mg}, 0.006 \mathrm{mmol})$ was added to a solution of $20(10.5 \mathrm{mg}, 0.032 \mathrm{mmol})$ in chloroform $(30 \mathrm{~mL})$. Oxygen was bubbled through the solution, and the reaction mixture was stirred under a halogen lamp for one hour. The solvent was removed in vacuo and purification by column chromatography (50\% EtOAc in hexanes) gave $22(8.3 \mathrm{mg}, 72.2 \%, 5: 1)$ as a colorless oil which was mixed with and could not be separated from $21 ; \mathrm{R}_{f} 0.17$ (50\% EtOAc in hexanes); HRMS (EI) calcd for $\mathrm{C}_{21} \mathrm{H}_{28} \mathrm{O}_{5}\left(\mathrm{M}^{+}\right): 360.193674$; found: 360.193560 . 


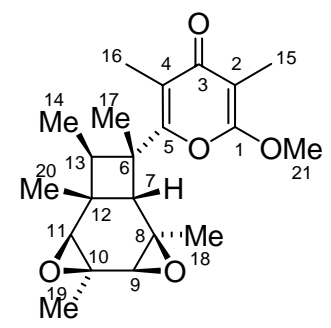

7

Elysiapyrone A (7): To a solution of $21(19 \mathrm{mg}, 0.053 \mathrm{mmol})$ in DCM (12 mL) at $0^{\circ} \mathrm{C}$ was added $\mathrm{RuCl}_{2}\left(\mathrm{PPh}_{3}\right)_{3}(1 \mathrm{mg}, 0.001 \mathrm{mmol})$. The reaction mixture was allowed to warm to room temperature with stirring. After $2 \mathrm{~h}$, the reaction mixture was concentrated in vacuo.

Purification by column chromatography (50\% EtOAc in hexanes) and HPLC (50-100\% EtOAc in hexanes over $30 \mathrm{~min}$, Dynamax-60A, Si 83-121-C) gave $7(12.9 \mathrm{mg}, 67.9 \%)$ as a white solid: $\mathrm{R}_{f} 0.17$ (50\% EtOAc in hexanes); IR (thin film) 2960, 2296, 1659, $1615 \mathrm{~cm}^{-1} ;{ }^{1} \mathrm{H}$ NMR (500 MHz): $\delta_{\mathrm{H}} 3.98(\mathrm{~s}, 3 \mathrm{H}), 3.18(\mathrm{~s}, 1 \mathrm{H}), 2.45-2.38(\mathrm{~m}, 2 \mathrm{H}), 2.27(\mathrm{~s}, 1 \mathrm{H}), 1.93(\mathrm{~s}, 3$ $\mathrm{H}), 1.89$ (s, $3 \mathrm{H}), 1.55(\mathrm{~s}, 3 \mathrm{H}), 1.38(\mathrm{~s}, 3 \mathrm{H}), 1.19(\mathrm{~m}, 6 \mathrm{H}), 1.02(\mathrm{~s}, 3 \mathrm{H}) ;{ }^{13} \mathrm{C} \mathrm{NMR}(125$ $\mathrm{MHz}): \delta_{\mathrm{C}} 182.6,166.2,163.3,119.0,105.5,62.3,61.3,60.6,59.4,56.9,54.1,45.0,44.0,35.9$, 23.6, 22.2, 20.5, 18.6, 11.4, 11.2, 7.6; HRMS (EI) calcd for $\mathrm{C}_{21} \mathrm{H}_{28} \mathrm{O}_{5}\left(\mathrm{M}^{+}\right): 360.193674$; found: 360.194119 .

\begin{tabular}{|c|c|c|c|c|}
\hline Position & ${ }^{13} \mathrm{C}$ (Lit.) & ${ }^{13} \mathrm{C}$ (Current) & ${ }^{1} \mathrm{H}$ (Lit.) & ${ }^{1} \mathrm{H}$ (Current) \\
\hline 1 & 163.2 & 163.3 & & \\
\hline 2 & 105.4 & 105.5 & & \\
\hline 3 & 182.5 & 182.6 & & \\
\hline 4 & 118.9 & 119.0 & & \\
\hline 5 & 166.1 & 166.2 & & \\
\hline 6 & 44.9 & 45.0 & & \\
\hline 7 & 54.0 & 54.1 & $2.27 \mathrm{~s}$ & $2.27 \mathrm{~s}$ \\
\hline 8 & 59.2 & 59.4 & & \\
\hline 9 & 61.1 & 61.3 & $3.18 \mathrm{~s}$ & $3.18 \mathrm{~s}$ \\
\hline 10 & 56.7 & 56.9 & & \\
\hline 11 & 62.1 & 62.3 & $2.39 \mathrm{~s}$ & $2.39 \mathrm{~s}$ \\
\hline 12 & 35.7 & 35.9 & & \\
\hline 13 & 43.8 & 44.0 & $2.43 q(7.5)$ & $2.45 \mathrm{q}(7.5)$ \\
\hline 14 & 20.3 & 20.5 & $1.19 \mathrm{~d}(7.5)$ & $1.19 \mathrm{~m}$ \\
\hline 15 & 7.4 & 7.6 & $1.93 \mathrm{~s}$ & $1.93 \mathrm{~s}$ \\
\hline 16 & 11.0 & 11.2 & $1.89 \mathrm{~s}$ & $1.89 \mathrm{~s}$ \\
\hline 17 & 23.4 & 23.6 & $1.38 \mathrm{~s}$ & $1.38 \mathrm{~s}$ \\
\hline 18 & 22.0 & 22.2 & $1.55 \mathrm{~s}$ & $1.55 \mathrm{~s}$ \\
\hline 19 & 11.2 & 11.4 & $1.20 \mathrm{~s}$ & $1.20 \mathrm{~s}$ \\
\hline 20 & 18.4 & 18.6 & $1.03 \mathrm{~s}$ & $1.02 \mathrm{~s}$ \\
\hline 21 & 60.4 & 60.6 & $3.98 \mathrm{~s}$ & $3.98 \mathrm{~s}$ \\
\hline
\end{tabular}




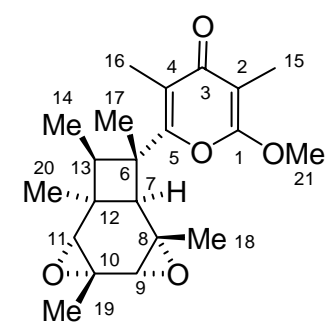

8

Elysiapyrone B (8): To a solution of crude $22(8.3 \mathrm{mg}, 0.023 \mathrm{mmol})$ in DCM $(12 \mathrm{~mL})$ at $0^{\circ} \mathrm{C}$ was added $\mathrm{RuCl}_{2}\left(\mathrm{PPh}_{3}\right)_{3}(1 \mathrm{mg}, 0.001 \mathrm{mmol})$. The reaction mixture was allowed to warm to room temperature with stirring. After two hours, the reaction mixture was concentrated in vacuo. Purification by column chromatography (50\% EtOAc in hexanes) and HPLC (50$100 \%$ EtOAc in hexanes over $30 \mathrm{~min}$, Dynamax-60A, Si 83-121-C) gave 8 (4.1 mg, 49.3\%) as a colorless oil: $\mathrm{R}_{f} 0.15$ (50\% EtOAc in hexanes); IR (thin film) 2924, 2466, 1657, 1613 $\mathrm{cm}^{-1}$; ${ }^{1} \mathrm{H}$ NMR $(500 \mathrm{MHz}): \delta_{\mathrm{H}} 4.05(\mathrm{~s}, 3 \mathrm{H}), 3.09(\mathrm{~s}, 1 \mathrm{H}), 2.94(\mathrm{~s}, 1 \mathrm{H}), 2.83(\mathrm{~s}, 1 \mathrm{H}), 2.53(\mathrm{q}$, $J=7.3 \mathrm{~Hz}, 1 \mathrm{H}), 2.02(\mathrm{~s}, 3 \mathrm{H}), 1.88(\mathrm{~s}, 3 \mathrm{H}), 1.60(\mathrm{~s}, 3 \mathrm{H}), 1.41(\mathrm{~s}, 3 \mathrm{H}), 1.38(\mathrm{~s}, 3 \mathrm{H}), 1.18(\mathrm{~s}$, $1 \mathrm{H}), 1.16(\mathrm{~m}, 1 \mathrm{H}) ;{ }^{13} \mathrm{C}$ NMR $(125 \mathrm{MHz}): \delta_{\mathrm{C}} 181.7,162.3,162.2,117.8,100.9,60.3,57.8$, 57.2, 56.8, 56.2, 46.9, 46.1, 44.3, 34.0, 26.2, 21.4, 21.1, 15.7, 10.8, 10.1, 7.1; HRMS (EI) calcd for $\mathrm{C}_{21} \mathrm{H}_{28} \mathrm{O}_{5}\left(\mathrm{M}^{+}\right)$: 360.193674 ; found: 360.193884 .

\begin{tabular}{|c|c|c|c|c|}
\hline Position & ${ }^{13} \mathrm{C}$ (Lit.) & ${ }^{13} \mathrm{C}$ (Current) & ${ }^{1} \mathrm{H}$ (Lit.) & ${ }^{1} \mathrm{H}$ (Current) \\
\hline 1 & 162.0 & 162.2 & & \\
\hline 2 & 100.9 & 100.9 & & \\
\hline 3 & 181.7 & 181.7 & & \\
\hline 4 & 117.8 & 117.8 & & \\
\hline 5 & 162.3 & 162.3 & & \\
\hline 6 & 44.3 & 44.3 & & $2.94 \mathrm{~s}$ \\
\hline 7 & 46.9 & 46.9 & $2.93 \mathrm{~s}$ & \\
\hline 8 & 56.2 & 56.2 & & $3.09 \mathrm{~s}$ \\
\hline 9 & 60.3 & 60.3 & $3.08 \mathrm{~s}$ & \\
\hline 10 & 56.7 & 56.8 & & $2.83 \mathrm{~s}$ \\
\hline 11 & 57.8 & 57.9 & $2.82 \mathrm{~s}$ & \\
\hline 12 & 34.0 & 34.1 & & $2.53 \mathrm{q}(7.3)$ \\
\hline 13 & 46.0 & 46.1 & $2.52 \mathrm{q}(6.8)$ & $1.16 \mathrm{~m}$ \\
\hline 14 & 10.0 & 10.1 & $1.16 \mathrm{~d}(6.8)$ & $1.88 \mathrm{~s}$ \\
\hline 15 & 7.1 & 7.1 & $1.86 \mathrm{~s}$ & $2.02 \mathrm{~s}$ \\
\hline 16 & 10.8 & 10.8 & $2.08 \mathrm{~s}$ & $1.38 \mathrm{~s}$ \\
\hline 17 & 15.7 & 15.7 & $1.37 \mathrm{~s}$ & $1.41 \mathrm{~s}$ \\
\hline 18 & 21.4 & 21.4 & $1.40 \mathrm{~s}$ & $1.60 \mathrm{~s}$ \\
\hline 19 & 21.0 & 21.1 & $1.58 \mathrm{~s}$ & $1.18 \mathrm{~s}$ \\
\hline 20 & 26.1 & 26.2 & $1.17 \mathrm{~s}$ & $4.05 \mathrm{~s}$ \\
\hline 21 & 57.3 & 57.3 & $4.03 \mathrm{~s}$ & \\
\hline
\end{tabular}

${ }^{1}$ Still, W. C.; Kahn, M.; Mitra, A. J. Org. Chem. 1978, 43, 2923-2925.

${ }^{2}$ Alaimo, P. J.; Peters, D. W.;Arnold, J.; Bergman, R. G. J. Chem. Ed. 2001, 78, 64.

${ }^{3}$ Parker, K. A.; Lim, Y. H. Org. Lett. 2004, 6, 161. 


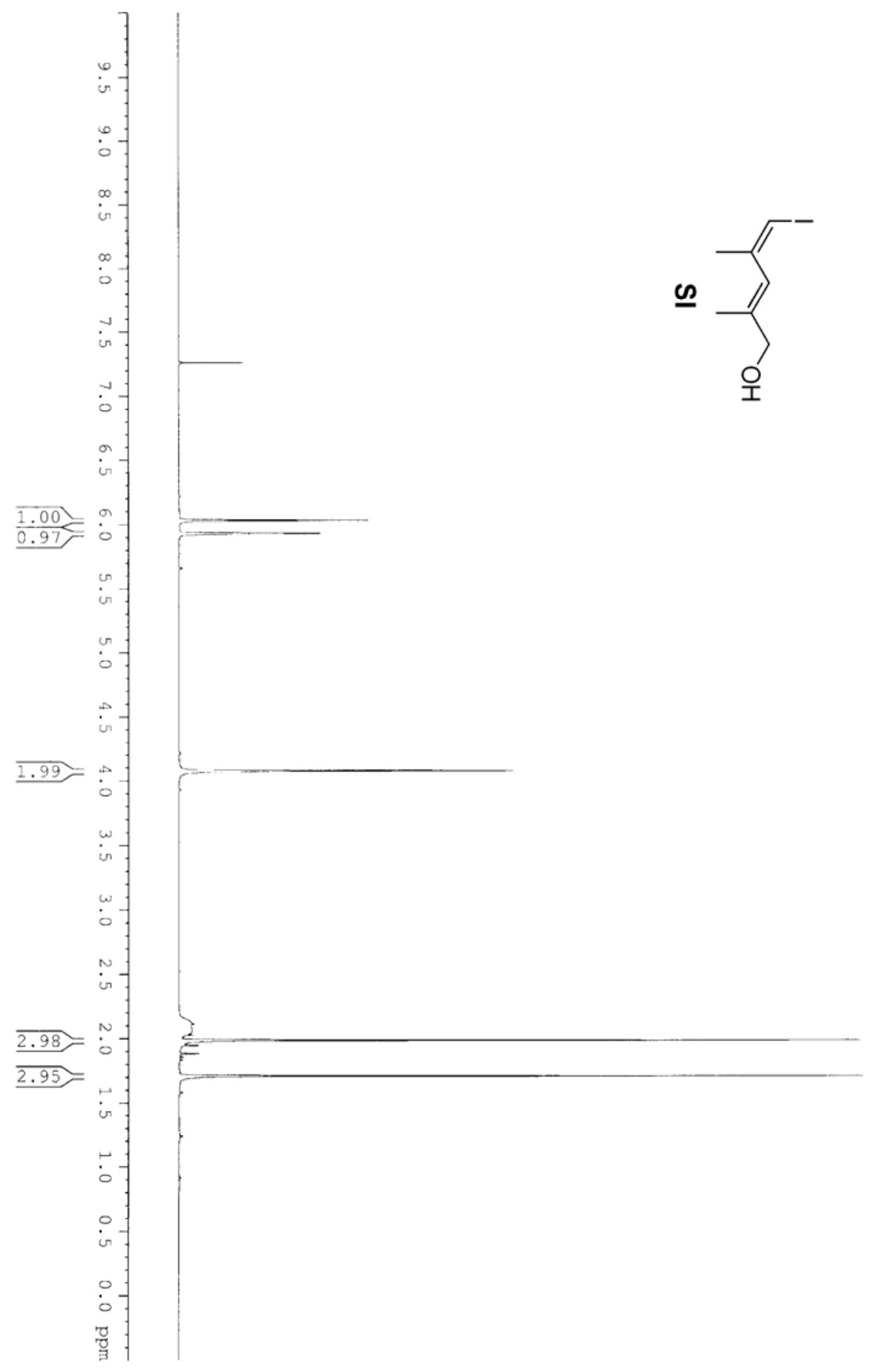




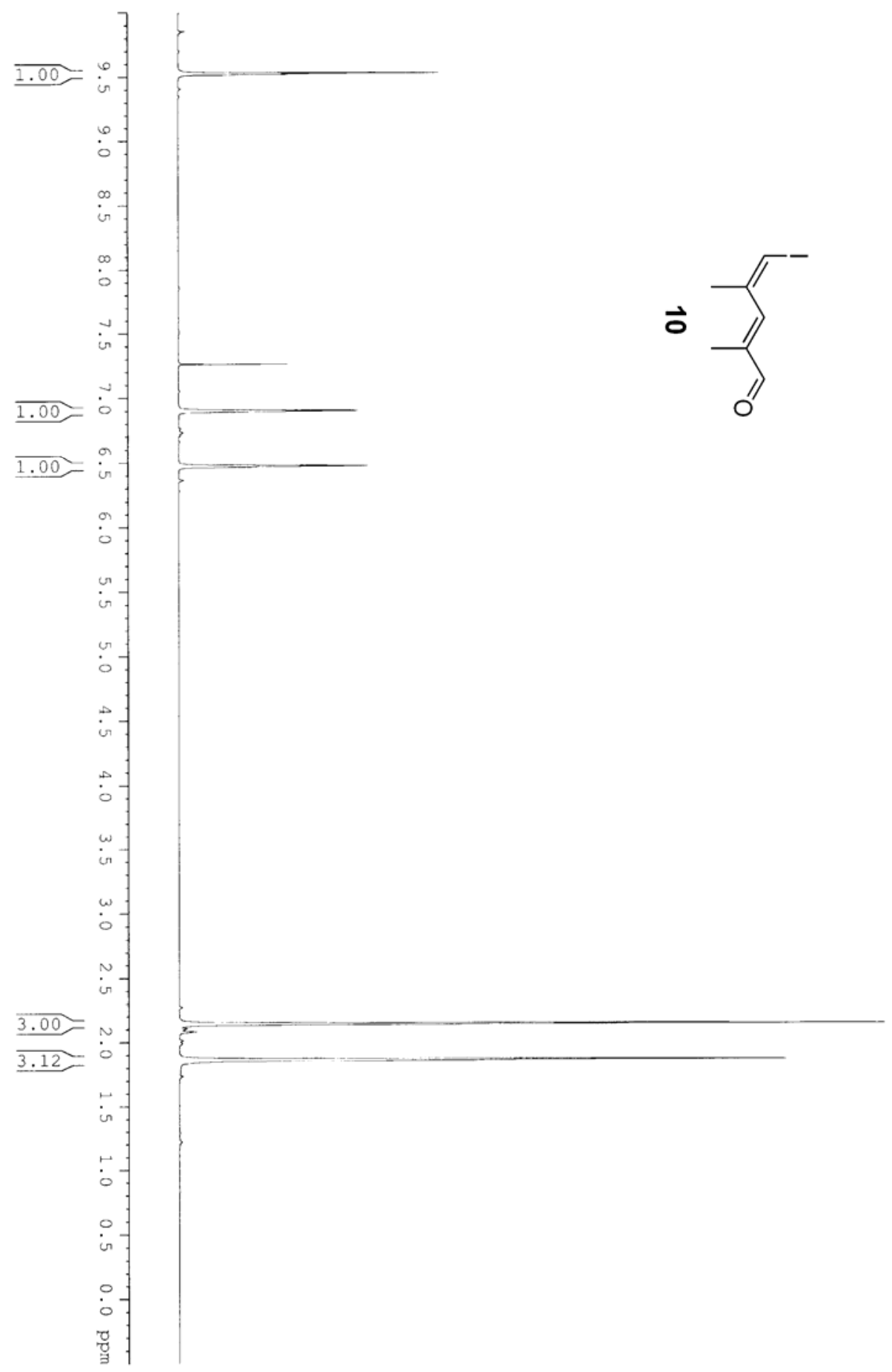




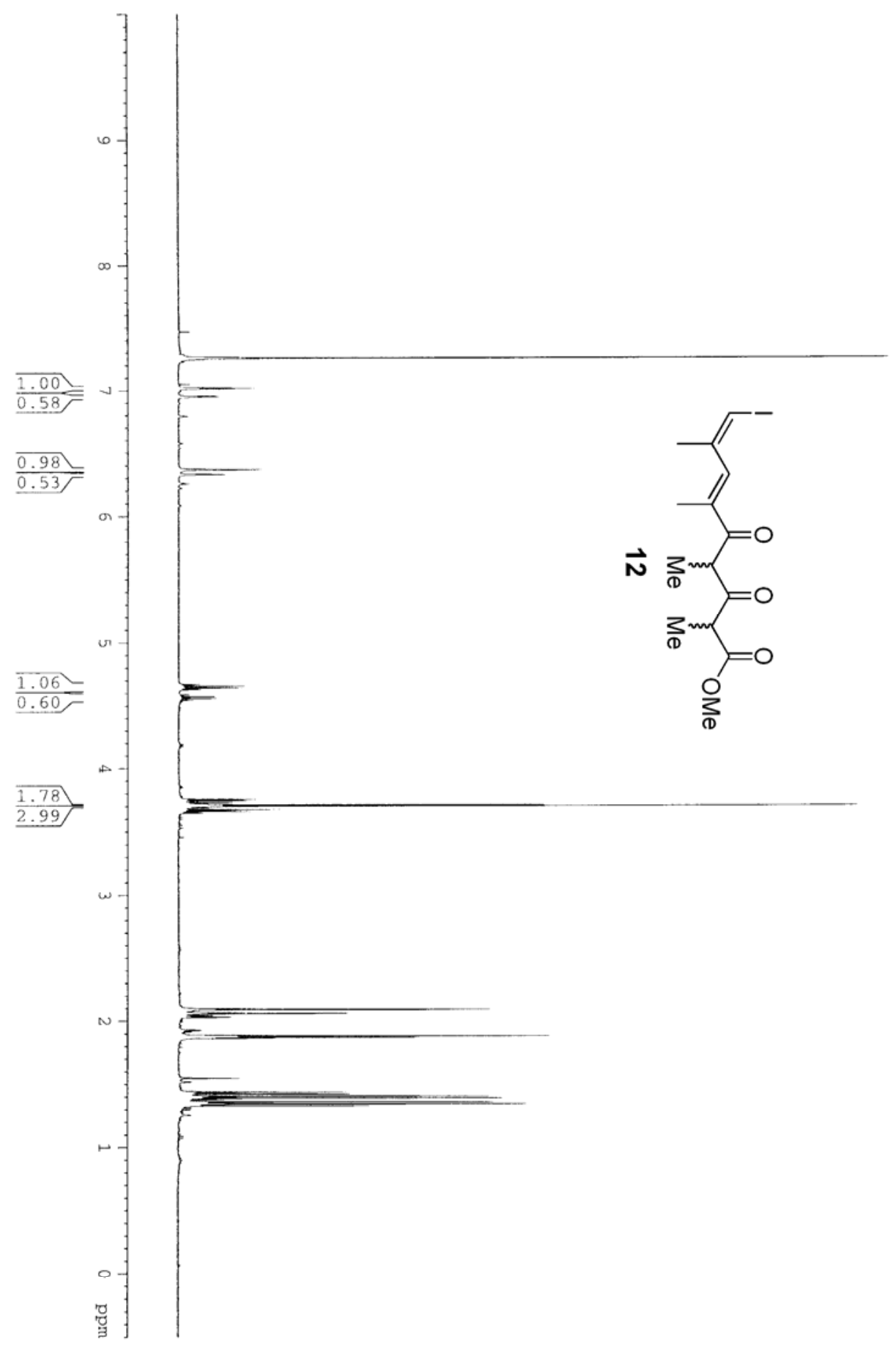




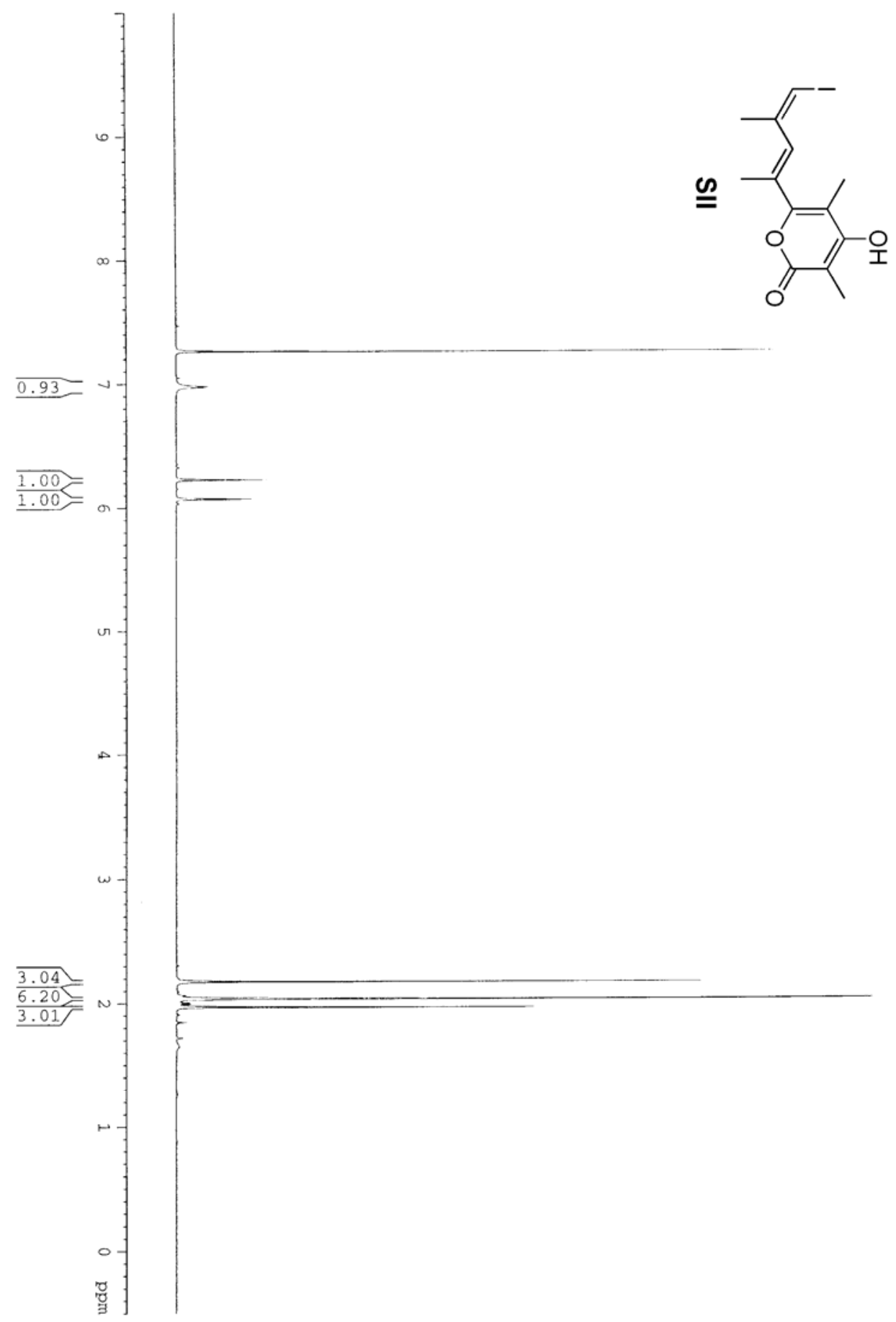




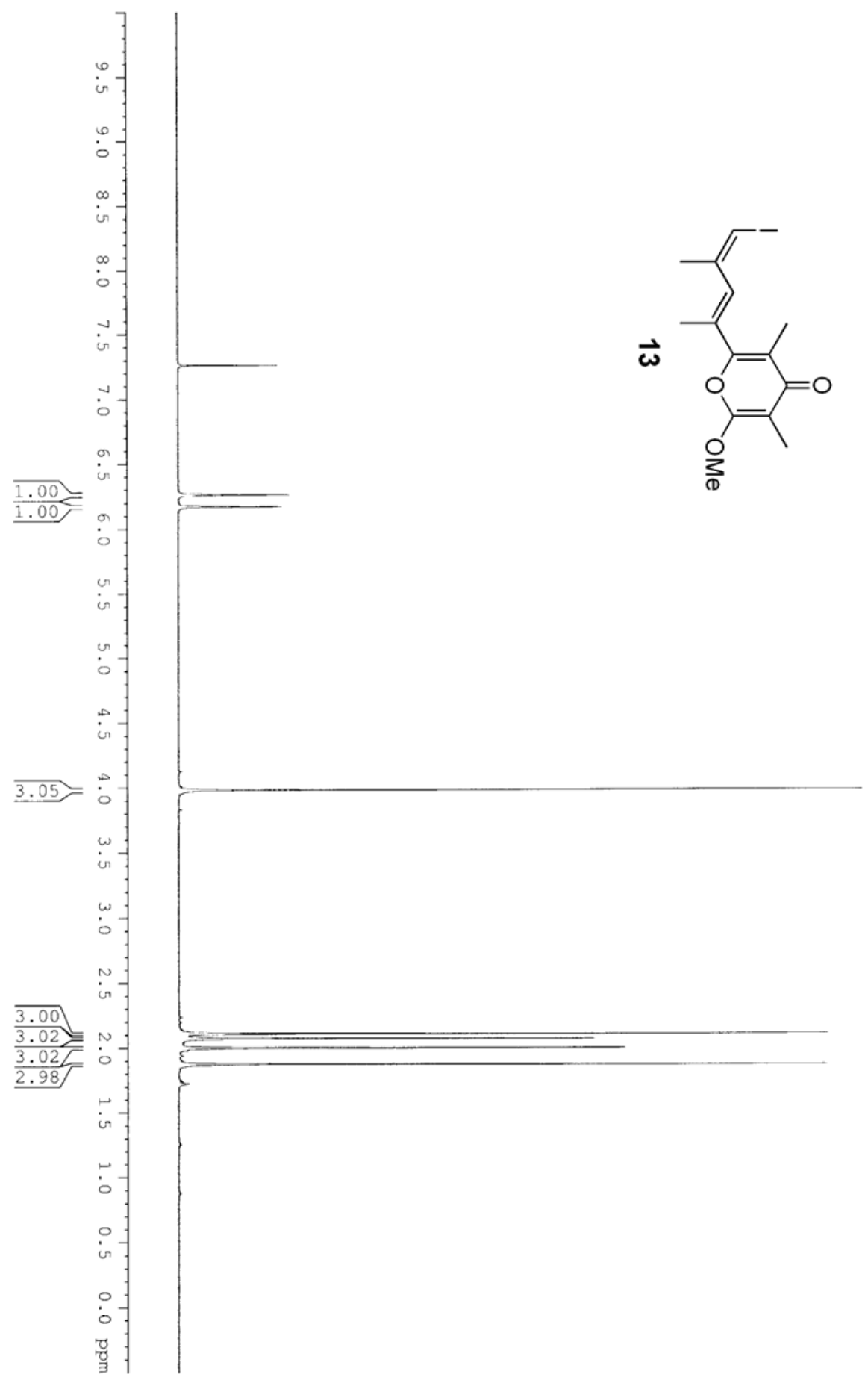




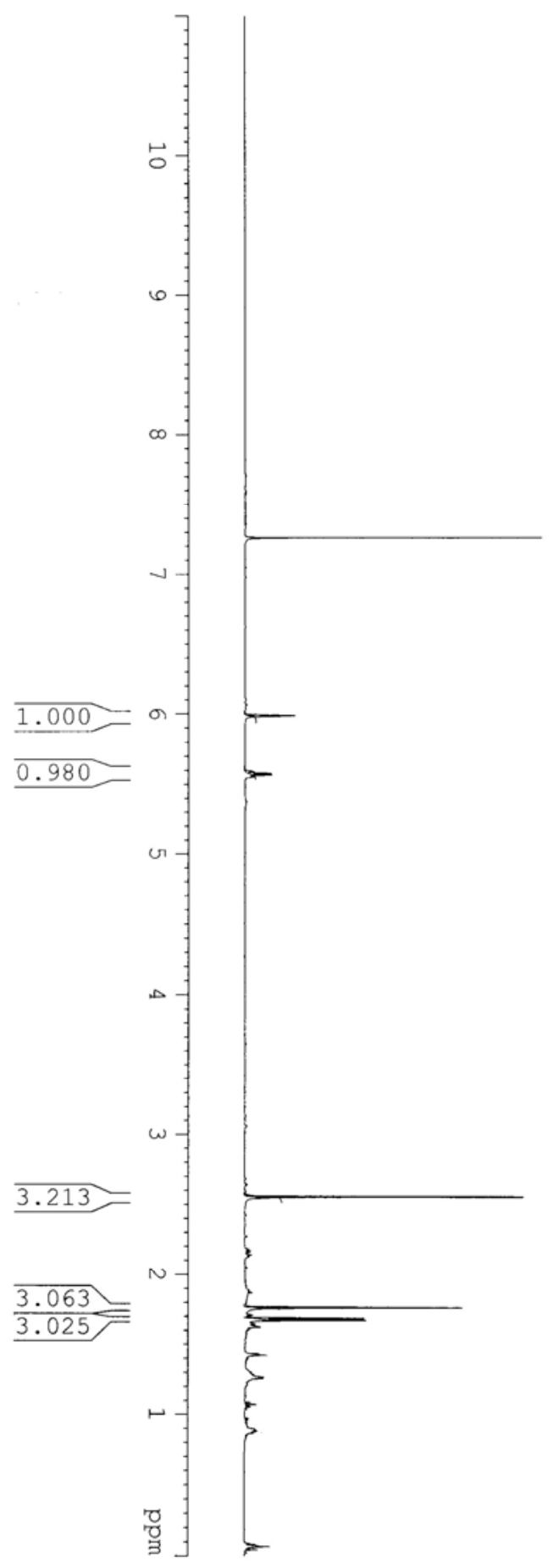




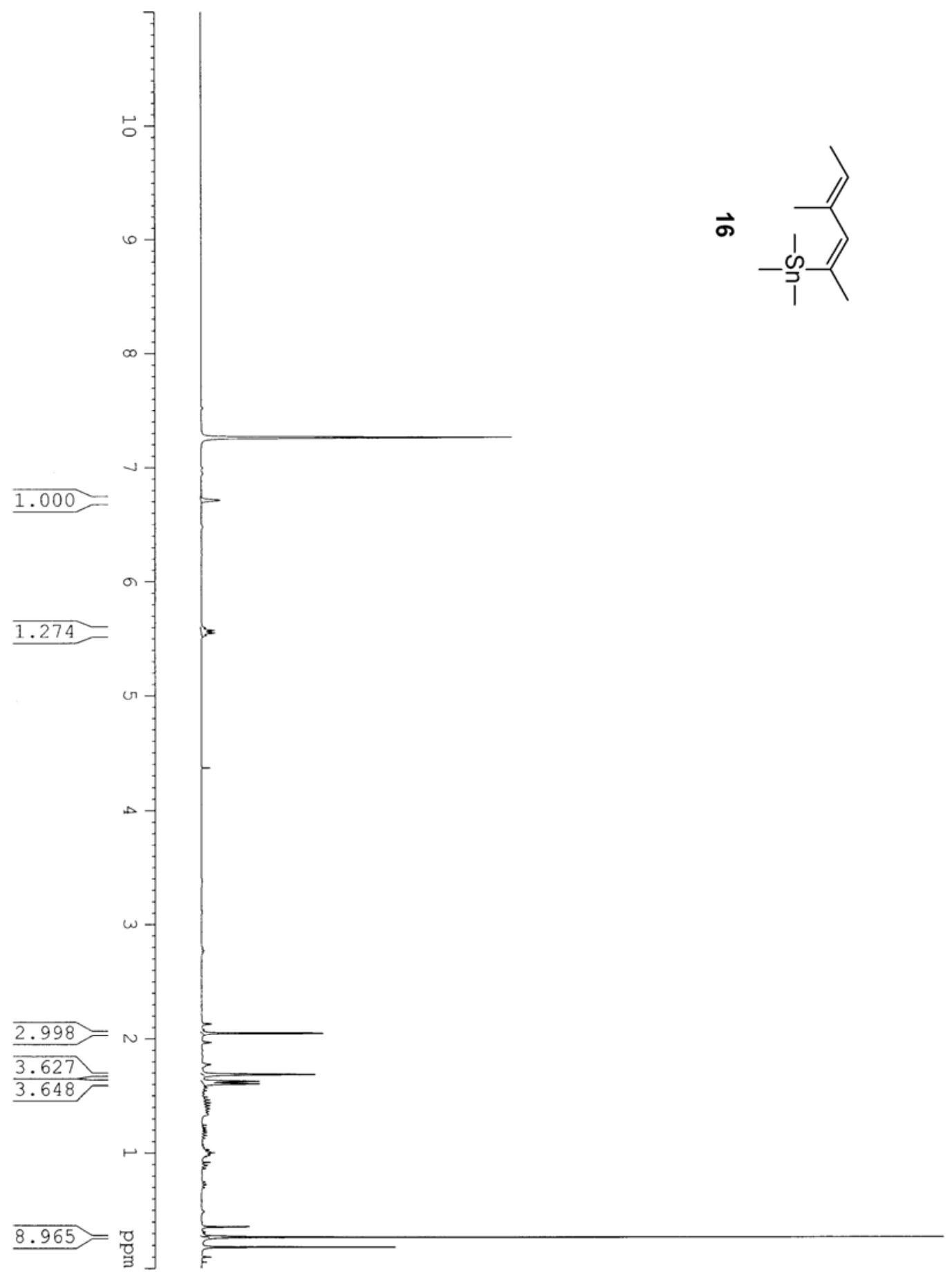




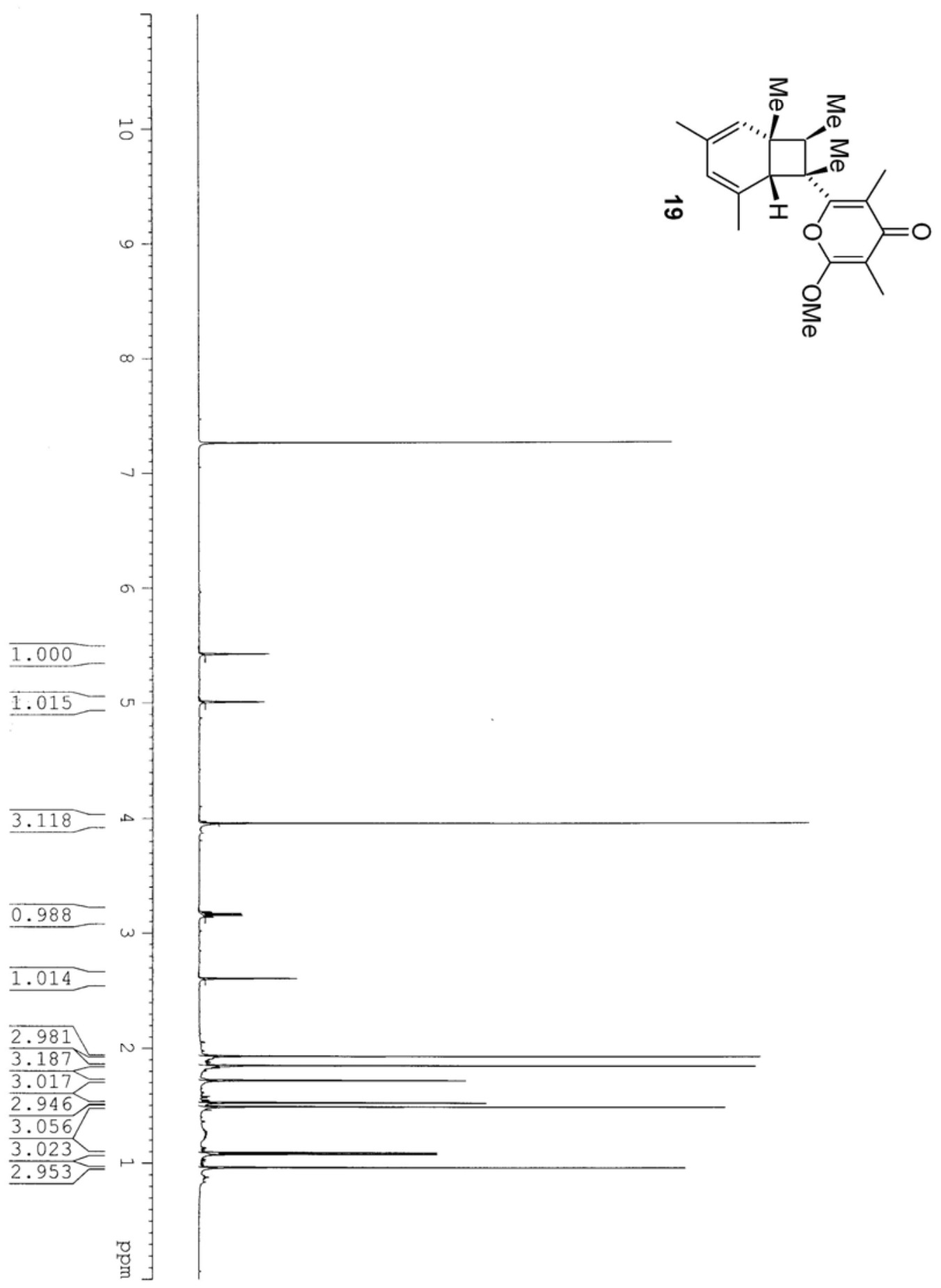




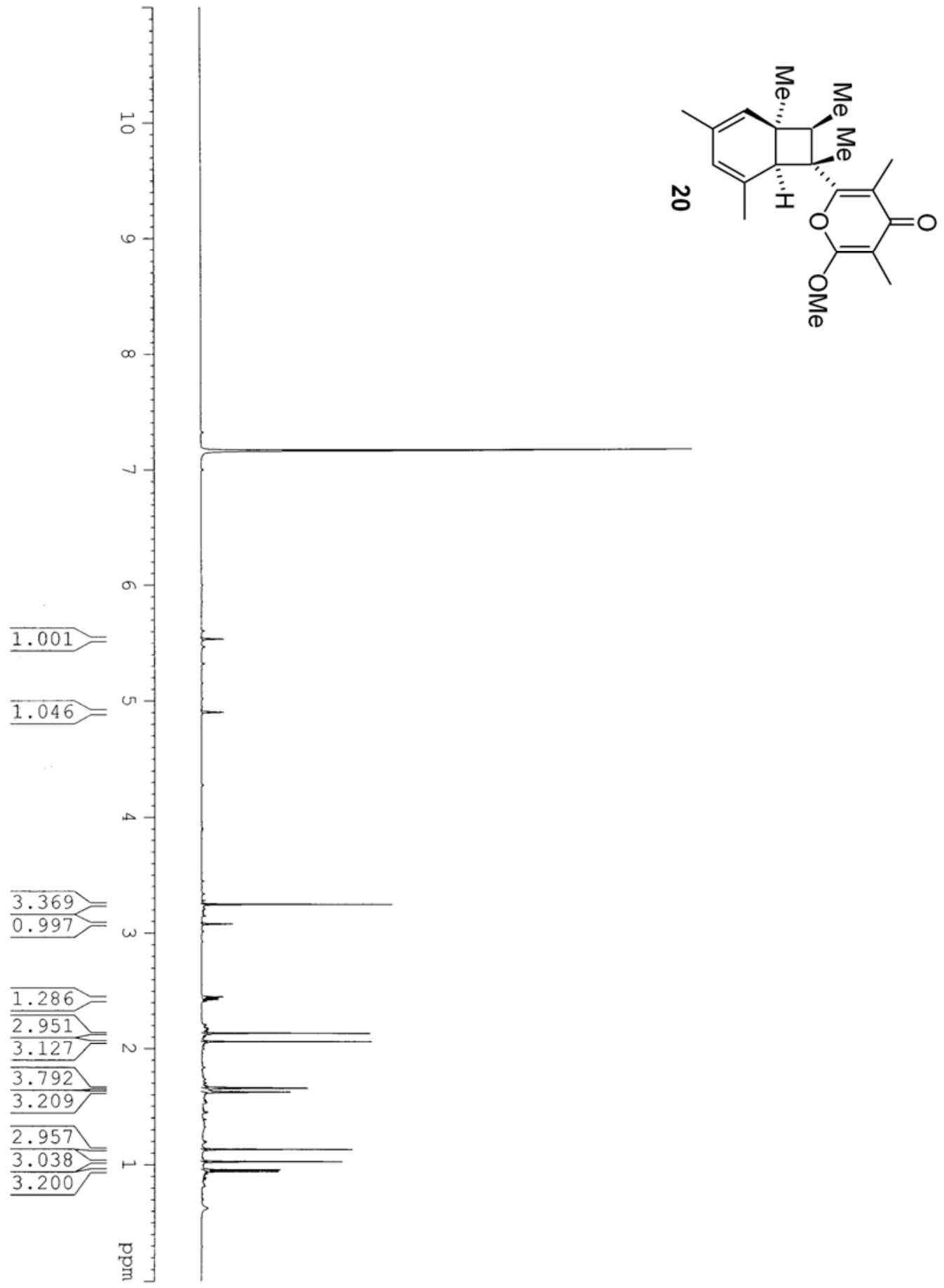




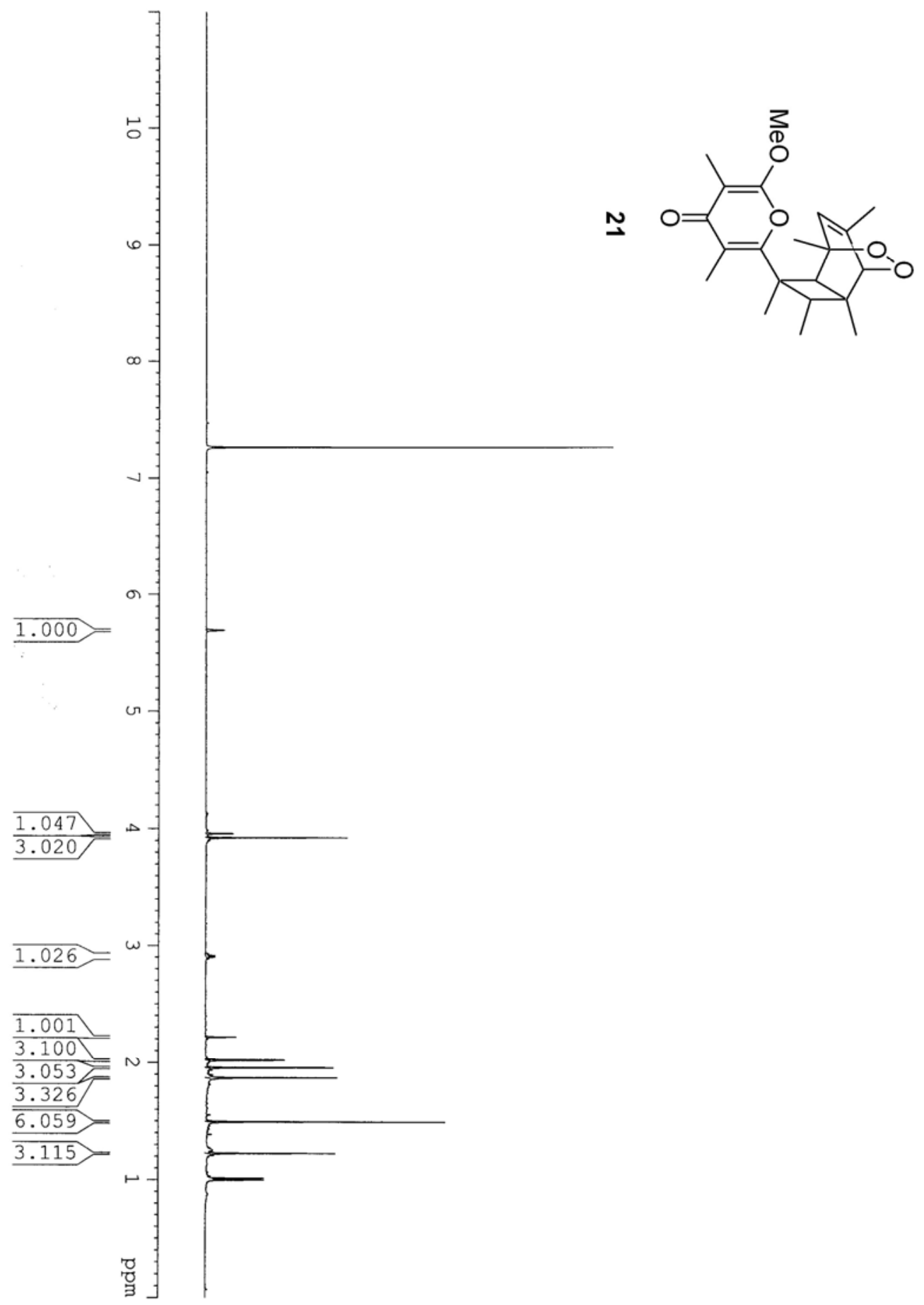




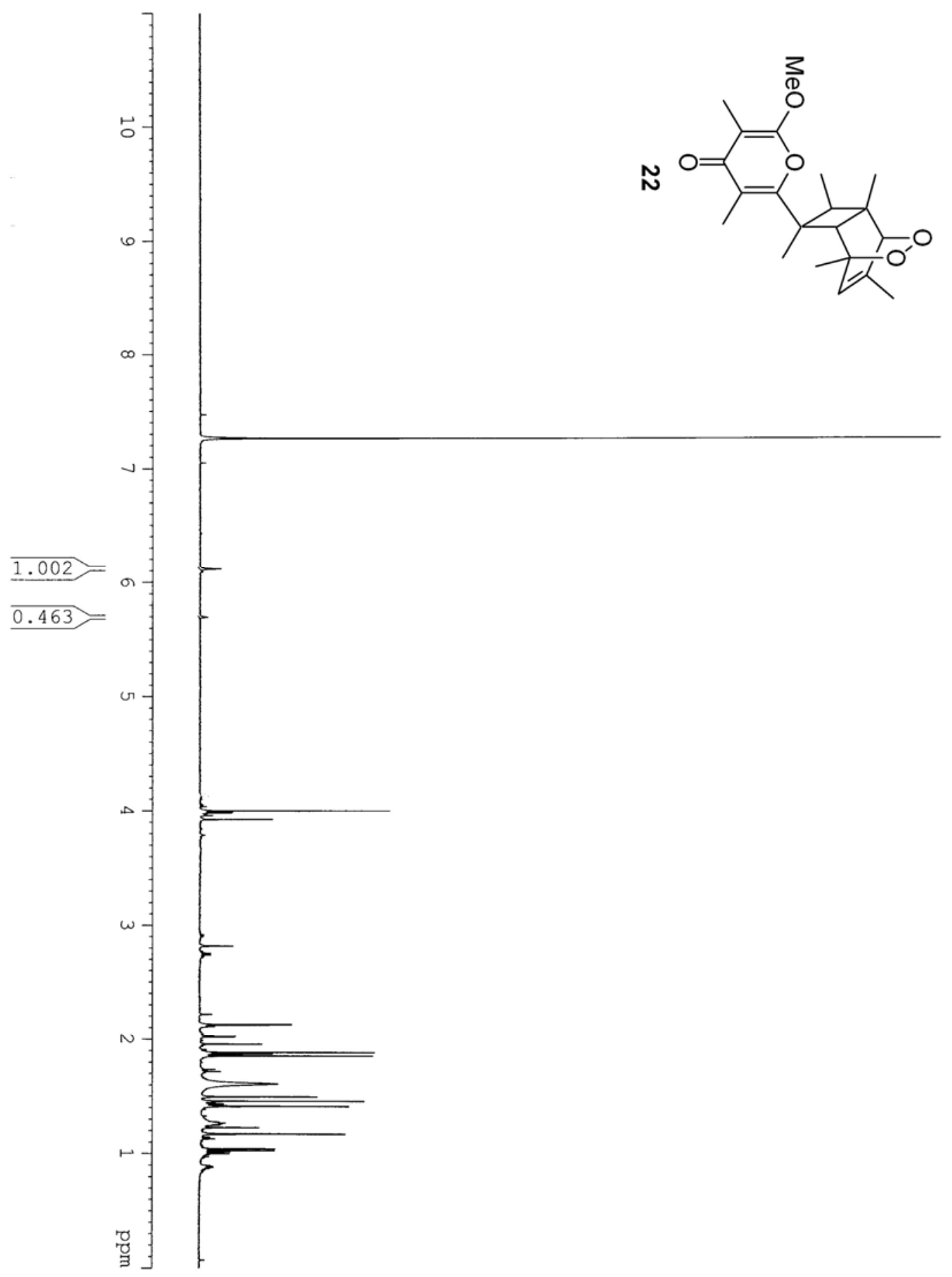



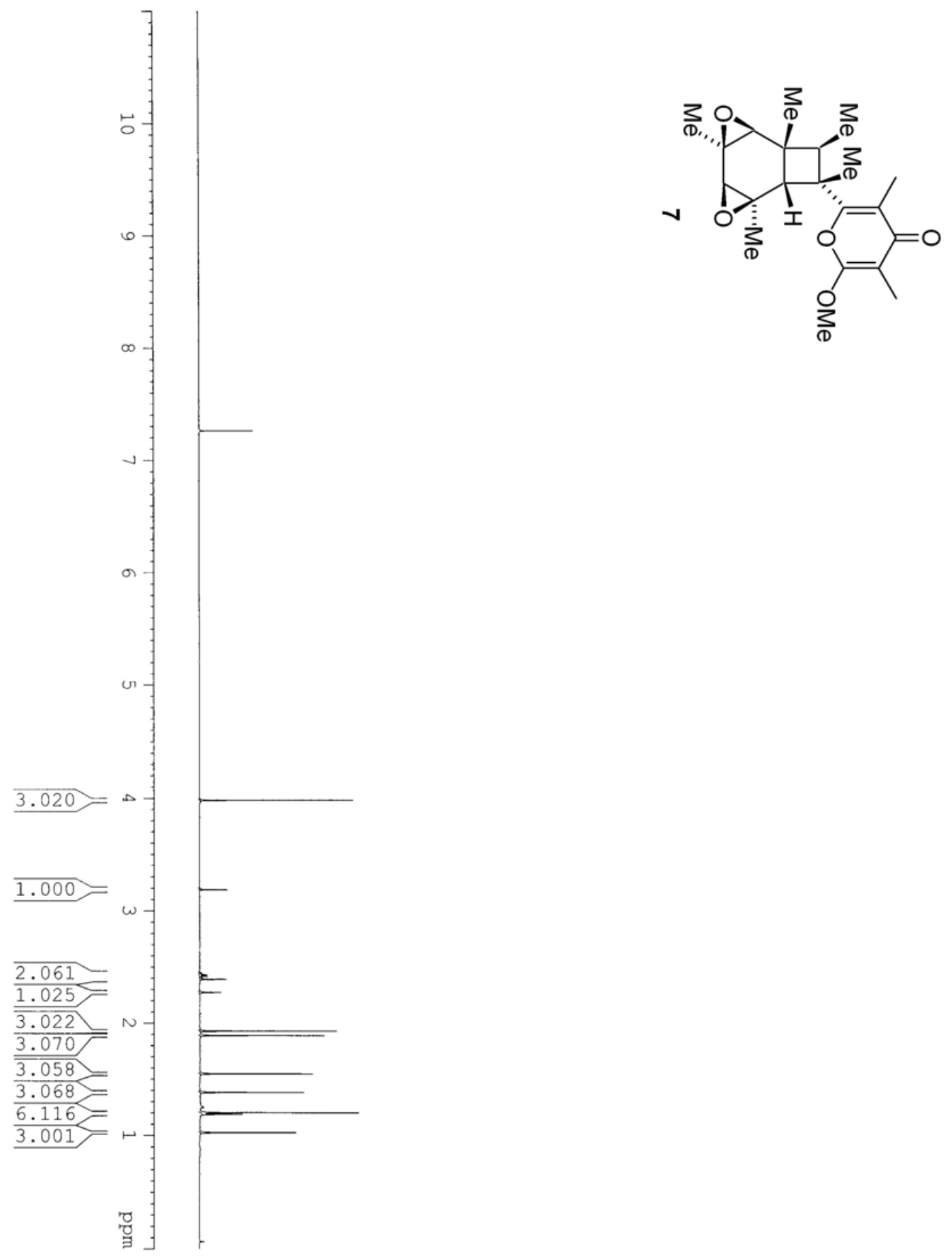


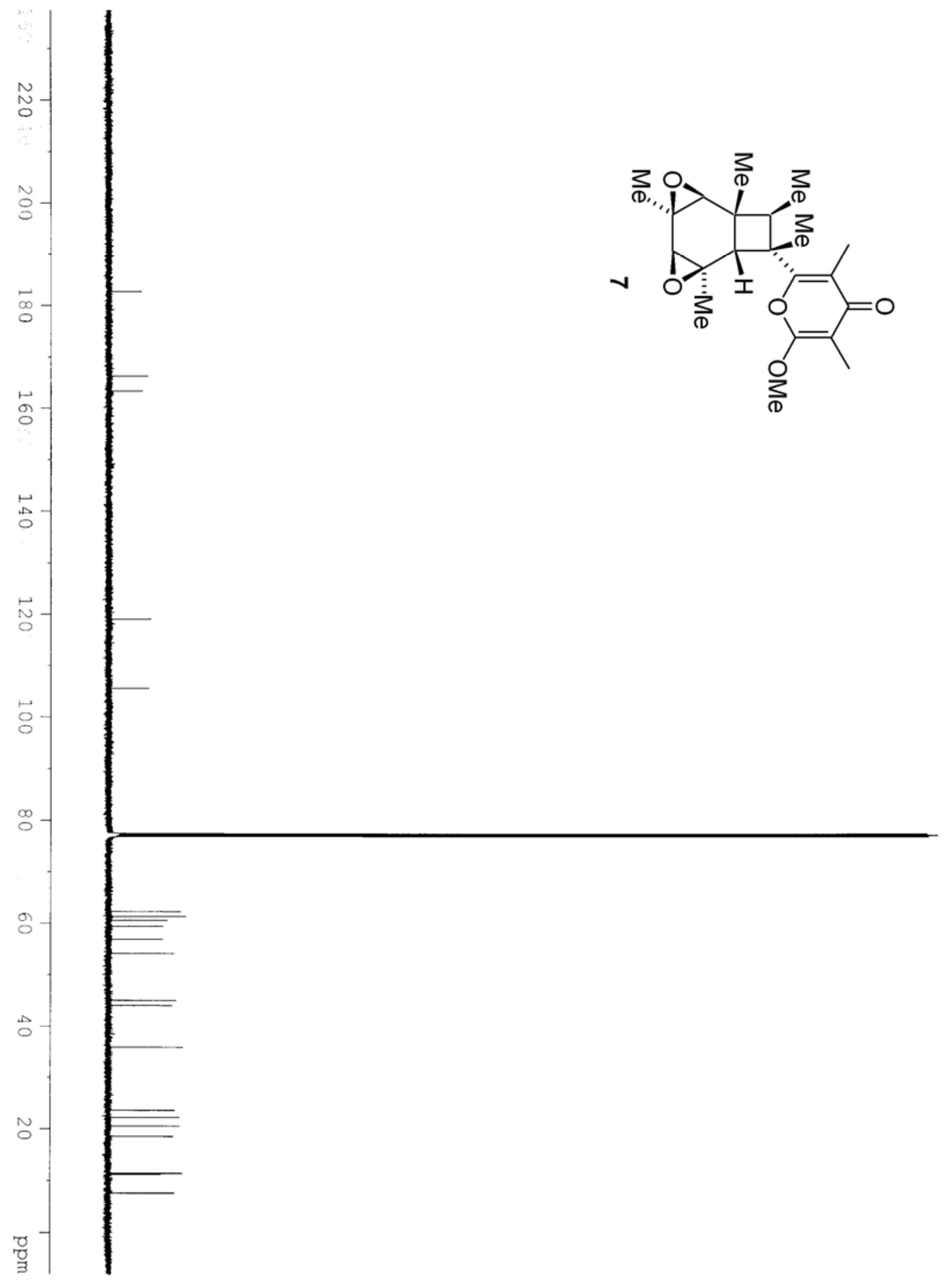




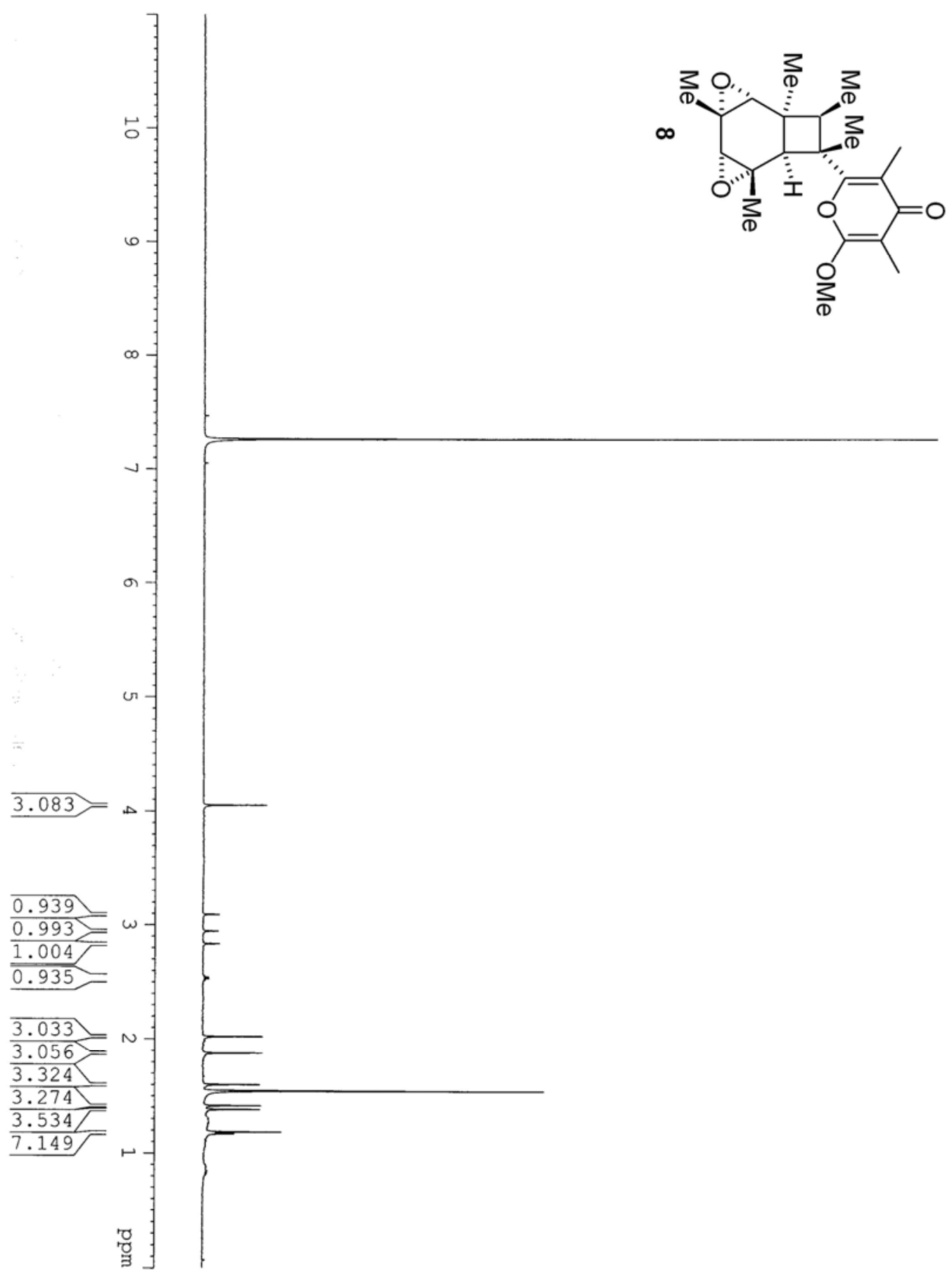




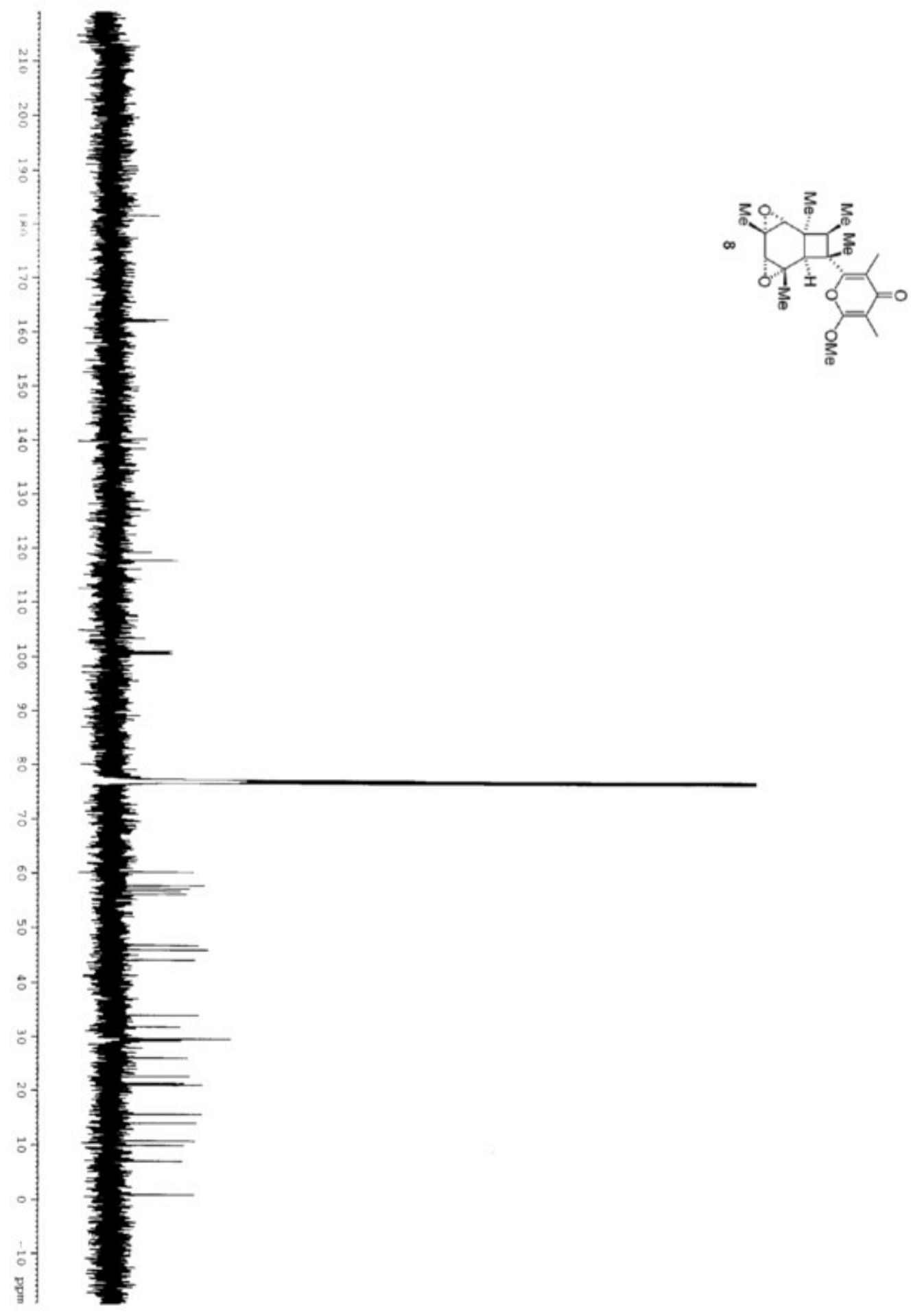

